\title{
CODAS METHOD FOR 2-TUPLE LINGUISTIC PYTHAGOREAN FUZZY MULTIPLE ATTRIBUTE GROUP DECISION MAKING AND ITS APPLICATION TO FINANCIAL MANAGEMENT PERFORMANCE ASSESSMENT
}

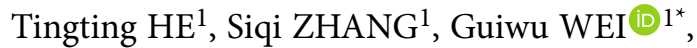 \\ Rui WANG ${ }^{1}$, Jiang $\mathrm{WU}^{2}$, Cun $\mathrm{WEI}^{2}$ \\ ${ }^{1}$ School of Business, Sichuan Normal University, Chengdu, 610101, P.R. China \\ ${ }^{2}$ School of Statistics, Southwestern University of Finance and Economics, Chengdu, 611130, P.R. China
}

Received 20 April 2019; accepted 20 October 2019

\begin{abstract}
Financial management performance evaluation (FMPE) has a significant effect on the identifying an investment chance. We can usually consider FMPE as a multiple attribute group decision making (MAGDM) issue, and the MAGDM method is needed to address it. Uncertainty may be one of the significant factors which could influence the process of MAGDM. In order to handle the uncertainty of group decision-making issues, MAGDM approaches along with 2-tuple linguistic Pythagorean fuzzy sets (2TLPFSs) have been designed. In this essay, CODAS method is extended to 2TLPFSs to tackle MAGDM issues. Linguistic variables and 2TLPFSs are also used to extend the CODAS method. An application of the presented 2-tuple linguistic Pythagorean fuzzy CODAS (2TLPF-CODAS) method to a case study of FMPE problem with 2-tuple linguistic Pythagorean fuzzy numbers (2TLPFNs) is given. To confirm the results, a comparative analysis between the fuzzy CODAS and 2TLPF-TODIM is performed. The results of the comparison illustrate that the presented 2TLPF-CODAS method offers effective and steady results.
\end{abstract}

Keywords: MAGDM, 2TLPFSs, CODAS method, 2TLPF-CODAS method, financial management performance evaluation.

JEL Classification: C43, C61, D81.

\section{Introduction}

The numbers 0 and 1 are used to deliver the "no" and "yes" of the depiction of the thing in the exact mathematical set, but there is often an ambiguous state in the depiction of the real world. On the basis of this, (Zadeh, 1965) presented the theory of fuzzy set which used the membership degree to describe things' ambiguity, but it fail to depict both support and opposition ideas (Wei, 2019a, 2019b; Wu et al., 2019a, 2019b; Wu et al., 2018). Thus,

*Corresponding author. E-mails: weiguiwu@163.com; weiguiwu1973@sicnu.edu.cn

This is an Open Access article distributed under the terms of the Creative Commons Attribution License (http://creativecommons. $\mathrm{org} / \mathrm{licenses} / \mathrm{by} / 4.0 /$ ), which permits unrestricted use, distribution, and reproduction in any medium, provided the original author and source are credited. 
Atanassov (1986) designed the intuitionistic fuzzy sets (IFSs) which could conquer this limitation. Reformat and Yager (2014) extended the IFSs with the Pythagorean fuzzy sets (PFSs), $u(x)$ and $v(x)$ should meet the new condition that $u^{2}(x)+v^{2}(x) \leq 1$, thus extending the description range of the IFSs. Until now, PFSs have widely applied in MADM and MAGDM (Khan et al., 2019; Zeb et al., 2019; Zhu et al., 2018). Based on the PFSs, Reformat and Yager (2014) presented a novel recommendation system which was collaborative oriented. Peng and Yang (2015) studied Pythagorean fuzzy numbers' division and subtraction algorithms. Garg (2017a) researched the confidence level's statistical concept into the PFSs. Ren et al. (2016) extended the Pythagorean fuzzy TODIM method on the basis of the prospect theory. Garg (2017b) improved scoring function calculation method for PFNs. Zeng et al. (2016) connected the distance measure with the PFSs. Li et al. (2018) defined some operators of Pythagorean Fuzzy Hamy Mean to address MAGDM issues. Zhang et al. (2017) combined the generalized Bonferroni mean with PFNs. Garg (2016) linked the PFSs with the Einstein operator. $\mathrm{Li}$ and $\mathrm{Lu}$ (2019) proposed some similarity and distance measures under PFSs. Wang et al. (2019b) designed the generalized Dice similarity measures for MAGDM with PFNs. Zhang (2016) extended the PFSs to the form of interval PFSs. Zhang and Jiang (2010) designed entropy for PFNs. Wei (2019c) defined the Hamacher power operators for PFNs. Zhang et al. (2016) presented a model which was about rough set under PFSs by means of multi-granular rough set. Deng et al. (2018a) gave the concept of 2TLPFSs and proposed various Hamy mean operations under 2TLPFSs. Deng et al. (2018) developed some Bonferroni mean operations under 2TLPFSs.

The CODAS method was defined by Keshavarz Ghorabaee et al. (2016). Panchal et al. (2017) employed integrated MCDM framework on the basis of AHP and CODAS method. Badi et al. (2018) made use of CODAS approach to choose the desalination plant's best location in Libya's northwestern coast. Ghorabaee et al. (2018) extended the CODAS method to fuzzy environment to choose the most desirable suppliers. Pamucar et al. (2018) introduced new CODAS method with linguistic Neutrosophic Numbers (LNN).

Therefore, the above research failed to concern about the MAGDM issue with 2TLPFNs in terms of CODAS approach. In this essay, we utilize the 2TLPFNs to expand the CODAS method to design a novel MAGDM method. An example is used to display the proposed model's applicability. To illustrate the 2TLPF-CODAS method's stability, we make a comparison between 2TLPF-CODAS method \& 2TLPF-TODIM method (Deng \& Gao, 2019). The calculating results demonstrate that the presented approach is stability and validity.

This paper's remainder is arranged subsequently. Some fundamental concepts of P2TLSs are given in Section 1. The CODAS method is built to handle MAGDM issues with 2TLPFNs in Section 2. A case study for FMPE is given to illustrate the presented approach in Section 3. In last section, the essay is made a conclusion.

\section{Preliminaries}

The fundamental concepts of 2-tuple linguistic sets (2TLSs) (Herrera \& Martinez, 2001a), Pythagorean fuzzy sets (PFSs) (Reformat \& Yager, 2014) and 2TLPFSs (Deng et al., 2018a) are given in this chapter. 


\subsection{TLSs}

Definition 1 (Herrera \& Martinez, 2001b). $S=\left\{s_{i} \mid i=0,1, \ldots, t\right\}$ is designed to be a linguistic term set (LTS) with odd integer. $s_{i}$ was employed to depict the possible value in a LTS, and the set $S$ could be depicted as:

$$
S=\left\{\begin{array}{l}
s_{0}=\text { extremely poor }, s_{1}=\text { very poor }, s_{2}=\text { poor }, s_{3}=\text { medium }, \\
s_{4}=\text { good, } s_{5}=\text { very good, } s_{6}=\text { extremely good. }
\end{array}\right\} .
$$

\subsection{PFSs}

The PFSs $A$ in a ordinary fixed set $X$ can be depicted underneath (Reformat \& Yager, 2014):

$$
A=\left\{\left\langle x, \mathrm{u}_{A}(x), v_{A}(x)\right\rangle \mid x \in X\right\},
$$

which $u_{A}(x)$ and $v_{A}(x)$ denoted the membership degree and the non-membership degree, which meet such condition: $0 \leq u_{A}(x) \leq 1,0 \leq v_{A}(x) \leq 1$ and $\left(u_{A}(x)\right)^{2}+\left(v_{A}(x)\right)^{2} \leq 1$.

\subsection{TLPFSs}

Deng et al. (2018a) gave the definition of 2TLPFSs.

Definition 1. (Deng et al., 2018a). Suppose that $P=\left\{p_{0}, p_{1}, \ldots, p_{t}\right\}$ is a LTSs with odd integer $t=1$. If $\left(s_{\alpha}(x), \varphi(x)\right),\left(s_{\beta}(x), \vartheta(x)\right)$ is defined for $s_{\alpha}(x), s_{\beta}(x) \in P, \varphi(x), \vartheta(x) \in[-0.5,0.5)$, where $\left(s_{\alpha}(x), \varphi(x)\right),\left(s_{\beta}(x), \vartheta(x)\right)$ depict the membership and non-membership by 2TLSs, then the definition of 2TLPFSs could be defined:

$$
P=\llbracket\left\langle x,\left\{\left(s_{\alpha}(x), \varphi(x)\right),\left(s_{\beta}(x), \vartheta(x)\right)\right\}\right\rangle \mid x \in X \rrbracket,
$$

where $0 \leq \Delta^{-1}\left(s_{\alpha}(x), \varphi(x)\right) \leq t, 0 \leq \Delta^{-1}\left(s_{\beta}(x), \vartheta(x)\right) \leq t$, $0 \leq\left(\Delta^{-1}\left(s_{\alpha}(x), \varphi(x)\right)\right)^{2}+\left(\Delta^{-1}\left(s_{\beta}(x), \vartheta(x)\right)\right)^{2} \leq t^{2}$.

In order to easy computation, $p=\left\{\left(s_{\alpha}, \varphi\right),\left(s_{\beta}, \vartheta\right)\right\}$ denotes the 2TLPFN.

Then, 2TLPFNs' score and accuracy function are shown as follows:

Definition 2. (Deng et al., 2018a). Let $p=\left\{\left(s_{\alpha}, \varphi\right),\left(s_{\beta}, \vartheta\right)\right\}$ be a 2TLPFN in $P$. Then the score and accuracy functions of $p$ are defined as follows:

$$
\begin{aligned}
& S(p)=\Delta\left\{\frac{t}{2}\left(1+\left(\frac{\Delta^{-1}\left(s_{\alpha}, \varphi\right)}{t}\right)^{2}-\left(\frac{\Delta^{-1}\left(s_{\beta}, \vartheta\right)}{t}\right)^{2}\right)\right\}, S(p) \in[0, t] \\
& \left.H(p)=\Delta\left\{t\left(\frac{\Delta^{-1}\left(s_{\alpha}, \varphi\right)}{t}\right)^{2}+\left(\frac{\Delta^{-1}\left(s_{\beta}, \vartheta\right)}{t}\right)^{2}\right)\right\}, H(p) \in[0, t] .
\end{aligned}
$$

Then, Deng et al. (2018a) gave some novel operations on the 2TLPFNs. 
Definition 3. (Deng et al., 2018a). Let $p_{1}=\left\{\left(s_{\alpha_{1}}, \varphi_{1}\right),\left(s_{\beta_{1}}, \vartheta_{1}\right)\right\}$ and $p_{2}=\left\{\left(s_{\alpha_{2}}, \varphi_{2}\right),\left(s_{\beta_{2}}, \vartheta_{2}\right)\right\}$ be two 2TLPFNs, then

(1) $p_{1} \oplus p_{2}=\left\{\Delta\left(t \sqrt{1-\left(1-\left(\frac{\Delta^{-1}\left(s_{\alpha_{1}}, \varphi_{1}\right)}{t}\right)^{2}\right)\left(1-\left(\frac{\Delta^{-1}\left(s_{\alpha_{2}}, \varphi_{2}\right)}{t}\right)^{2}\right)}\right)\right.$,

$$
\left.\Delta\left(t\left(\frac{\Delta^{-1}\left(s_{\beta_{1}}, \vartheta_{1}\right)}{t} \bullet \frac{\Delta^{-1}\left(s_{\beta_{2}}, \vartheta_{2}\right)}{t}\right)\right)\right\}
$$

(2) $p_{1} \otimes p_{2}=\left\{\Delta\left(t\left(\frac{\Delta^{-1}\left(s_{\alpha_{1}}, \varphi_{1}\right)}{t} \bullet \frac{\Delta^{-1}\left(s_{\alpha_{2}}, \varphi_{2}\right)}{t}\right)\right)\right.$,

$$
\left.\Delta\left(t \sqrt{1-\left(1-\left(\frac{\Delta^{-1}\left(s_{\beta_{1}}, \vartheta_{1}\right)}{t}\right)^{2}\right)\left(1-\left(\frac{\Delta^{-1}\left(s_{\beta_{2}}, \vartheta_{2}\right)}{t}\right)^{2}\right)}\right)\right\}
$$

(3) $\lambda p_{1}=\left\{\Delta\left(t \sqrt{1-\left(1-\left(\frac{\Delta^{-1}\left(s_{\alpha_{1}}, \varphi_{1}\right)}{t}\right)^{2}\right)^{\lambda}}\right), \Delta\left(t\left(\frac{\Delta^{-1}\left(s_{\beta_{1}}, \vartheta_{1}\right)}{t}\right)^{\lambda}\right)\right\}$

(4) $\left(p_{1}\right)^{\lambda}=\left\{\Delta\left(t\left(\frac{\Delta^{-1}\left(s_{\alpha_{1}}, \varphi_{1}\right)}{t}\right)^{\lambda}\right), \Delta\left(t \sqrt{1-\left(1-\left(\frac{\Delta^{-1}\left(s_{\beta_{1}}, \vartheta_{1}\right)}{t}\right)^{2}\right)^{\lambda}}\right)\right\}$.

Definition 4. (Deng \& Gao, 2019). Let $p_{1}=\left\{\left(s_{\alpha_{1}}, \varphi_{1}\right),\left(s_{\beta_{1}}, \vartheta_{1}\right)\right\}$ and $p_{2}=\left\{\left(s_{\alpha_{2}}, \varphi_{2}\right),\left(s_{\beta_{2}}, \vartheta_{2}\right)\right\}$ be two 2TLPFNs, then the normalized Hamming distance $H D$ is designed:

$$
H D\left(p_{1}, p_{2}\right)=\frac{1}{2 t}\left(\left|\Delta^{-1}\left(s_{\alpha_{1}}, \varphi_{1}\right)-\Delta^{-1}\left(s_{\alpha_{2}}, \varphi_{2}\right)\right|+\left|\Delta^{-1}\left(s_{\beta_{1}}, \vartheta_{1}\right)-\Delta^{-1}\left(s_{\beta_{2}}, \vartheta_{2}\right)\right|\right) .
$$

Definition 5. Let $p_{1}=\left\{\left(s_{\alpha_{1}}, \varphi_{1}\right),\left(s_{\beta_{1}}, \vartheta_{1}\right)\right\}$ and $p_{2}=\left\{\left(s_{\alpha_{2}}, \varphi_{2}\right),\left(s_{\beta_{2}}, \vartheta_{2}\right)\right\}$ be two 2TLPFNs, then the normalized Hamming distance $E D$ is designed:

$$
E D\left(p_{1}, p_{2}\right)=\sqrt{\frac{1}{2}\left(\left(\frac{\left|\Delta^{-1}\left(s_{\alpha_{1}}, \varphi_{1}\right)-\Delta^{-1}\left(s_{\alpha_{2}}, \varphi_{2}\right)\right|}{t}\right)^{2}+\left(\frac{\left|\Delta^{-1}\left(s_{\beta_{1}}, \vartheta_{1}\right)-\Delta^{-1}\left(s_{\beta_{2}}, \vartheta_{2}\right)\right|}{t}\right)^{2}\right)}
$$




\section{The CODAS method for MAGDM with 2TLPFNs}

The subsequently assumptions or notations are utilized to denote the MAGDM issues with 2TLPFNs. Assume $A=\left\{A_{1}, A_{2}, \cdots, A_{m}\right\}$ be some chosen alternatives and $G=\left\{G_{1}, G_{2}, \cdots, G_{n}\right\}$ be some designed attributes with weight vector $w=\left(w_{1}, w_{2}, \cdots, w_{n}\right)$, where $w_{j} \in[0,1]$, $\sum_{j=1}^{n} w_{j}=1$, and some experts $E=\left\{E_{1}, E_{2}, \cdots, E_{q}\right\}$ with weight vector $v=\left(v_{1}, v_{2}, \cdots, v_{q}\right)$, where $v_{k} \in[0,1], k=1,2, \cdots, q, \sum_{k=1}^{q} v_{k}=1$. Suppose that there are $n$ qualitative attribute $G=\left\{G_{1}, G_{2}, \cdots, G_{n}\right\}$ and their values are assessed by each expert and depicted as linguistic expressions $l_{i j}^{k}(i=1,2, \cdots, m, j=1,2, \cdots, n, k=1,2, \cdots, q)$ in Table 1 .

Table 1. Linguistic variables and their 2TLPFNs

\begin{tabular}{|l|l|}
\hline \multicolumn{1}{|c|}{ Linguistic variable } & \multicolumn{1}{c|}{ 2TLPFNs } \\
\hline Very low $(\mathrm{VL})$ & $\left.\left\{\left(\mathrm{s}_{0}, 0\right),\left(\mathrm{s}_{6}, 0\right)\right)\right\}$ \\
\hline Low $(\mathrm{L})$ & $\left\{\left(\mathrm{s}_{1}, 0\right),\left(\mathrm{s}_{5}, 0\right)\right\}$ \\
\hline Medium low $(\mathrm{ML})$ & $\left\{\left(\mathrm{s}_{2}, 0\right),\left(\mathrm{s}_{4}, 0\right)\right\}$ \\
\hline Medium $(\mathrm{M})$ & $\left\{\left(\mathrm{s}_{3}, 0\right),\left(\mathrm{s}_{3}, 0\right)\right\}$ \\
\hline Medium high $(\mathrm{MH})$ & $\left\{\left(\mathrm{s}_{4}, 0\right),\left(\mathrm{s}_{2}, 0\right)\right\}$ \\
\hline High $(\mathrm{H})$ & $\left\{\left(\mathrm{s}_{5}, 0\right),\left(\mathrm{s}_{1}, 0\right)\right\}$ \\
\hline Very high $(\mathrm{VH})$ & $\left\{\left(\mathrm{s}_{6}, 0\right),\left(\mathrm{s}_{0}, 0\right)\right\}$ \\
\hline
\end{tabular}

Then, an extended CODAS method with 2TLPFNs is proposed to tackle the MAGDM issues. The calculating steps are involved as follows:

Step 1. Switch the linguistic information $l_{i j}^{k}$ into 2TLPFNs $r_{i j}^{k}=\left\{\left(s_{\phi_{i j}^{k}}, \varphi_{i j}^{k}\right),\left(s_{\theta_{i j}^{k}}, \vartheta_{i j}^{k}\right)\right\}$.

Step 2. According to 2TLPFN $r_{i j}^{k}=\left\{\left(s_{\phi_{i j}^{k}}, \varphi_{i j}^{k}\right),\left(s_{\theta_{i j}^{k}}, \vartheta_{i j}^{k}\right)\right\}$ and 2TLPFWA operator (Deng et al., 2018b), the experts' individual evaluations can be fused into the collective 2TLPFNs $r_{i j}=\left\{\left(s_{\phi_{i j}}, \varphi_{i j}\right),\left(s_{\theta_{i j}}, \vartheta_{i j}\right)\right\}$.

$$
R=\left[r_{i j}\right]_{m \times n}=\left[\begin{array}{cccc}
r_{11} & r_{12} & \ldots & r_{1 n} \\
r_{21} & r_{22} & \ldots & r_{2 n} \\
\vdots & \vdots & \vdots & \vdots \\
r_{m 1} & r_{m 2} & \ldots & r_{m n}
\end{array}\right] ;
$$

$$
\begin{aligned}
& r_{i j}=\operatorname{2TLPFWA}\left(r_{i j}^{1}, r_{i j}^{2} \cdots, r_{i j}^{q}\right)=\bigoplus_{k=1}^{q} \eta_{k} r_{i j}^{k}= \\
& \left\{\Delta\left(\sqrt{1-\prod_{k=1}^{q}\left(1-\left(\frac{\Delta^{-1}\left(s_{\phi_{i j}^{k}}, \varphi_{i j}^{k}\right)}{t}\right)^{2}\right)^{\eta_{k}}}\right), \Delta\left(t \prod_{k=1}^{q}\left(\frac{\Delta^{-1}\left(s_{\theta_{i j}^{k}}, \vartheta_{i j}^{k}\right)}{t}\right)\right)\right\} .
\end{aligned}
$$


Step 3. Calculate the 2 TLPF weighted matrix.

$$
t_{i j}=w_{j} \otimes r_{i j},
$$

where $w_{j}$ means the attribute weight of $G_{j}$, and $0 \leq w_{j} \leq 1, \sum_{j=1}^{n} w_{j}=1$.

Step 4. Get the negative ideal solution with score and accuracy functions of 2TLPFNs (if score functions are equal, the accuracy functions are chosen to rank the 2TLPFNs):

$$
\begin{aligned}
& N I S=\left[N I S_{j}\right]_{1 \times n} ; \\
& N I S_{j}=\min _{i} S\left(t_{i j}\right) .
\end{aligned}
$$

Step 5. Determine the weighted $E D_{i}$ and $H D_{i}$ :

$$
\begin{aligned}
E D_{i} & =\sum_{j=1}^{n} E D\left(t_{i j}, N I S_{j}\right) ; \\
H D_{i} & =\sum_{j=1}^{n} H D\left(t_{i j}, N I S_{j}\right) .
\end{aligned}
$$

Step 6. Build the relative assessment matrix $R A$ in subsequently equations:

$$
\begin{gathered}
R A=\left[h_{i k}\right]_{m \times m} ; \\
h_{i k}=\left(E D_{i}-E D_{k}\right)+\left(g\left(E D_{i}-E D_{k}\right) \times\left(H D_{i}-H D_{k}\right)\right),
\end{gathered}
$$

where $k \in\{1,2, \ldots, m\}$ and $g$ means an important function which could be designed:

$$
g(\theta)=\left\{\begin{array}{ll}
1 & \text { if }|\theta| \geq \tau \\
0 & \text { if }|\theta|<\tau
\end{array},\right.
$$

where $\tau \in[0.01,0.05]$ given by DMs. In current study, $\tau=0.02$.

Step 7. Derive the $A S_{i}$ by Eq. (28).

$$
A S_{i}=\sum_{k=1}^{m} h_{i k}
$$

Step 8. All the alternatives can be ranked on the basis of the computing results of $A S_{i}$. The best alternative has the highest assessment score.

\section{Case study and comparative analysis}

The financial management performance issue is a classical MAGDM issue (Erdogan et al., 2019; Lu et al., 2019; Roy et al., 2019; Tabatabaei et al., 2019; Wang et al., 2019a; Wang, 2019; Wei et al., 2019a, 2019b). In this chapter, we shall give a case study of the financial management performance to choose the most desirable enterprise which has the best financial performance by utilizing CODAS method with 2TLPFNs. Assume that an enterprise identified an investment chance with enterprise financial performances, and in order to maximize the expected profit, we need to determine the enterprise financial performances of the five 
enterprises so as to choose the optimal one. The investment company has to make a decision in terms of the subsequently four benifical attributes: $G_{1}$ is the enterprise innovation ability; $G_{2}$ is the enterprise resource utilization capability; $G_{3}$ is the internal process; $G_{4}$ is the corporate credit rating. There are five potential enterprises $A_{i}(i=1,2,3,4,5)$ to be assessed by using linguistic variables which are listed in Table 1 . These linguistic variables are given by the invited DMs $D_{k}(k=1,2,3)$ (whose weighting vector $v=(0.20,0.50,0.30)$ ) within the mentioned attributes (whose weighting vector $\omega=(0.20,0.30,0.40,0.10)^{T}$ ), and set up three decision matrixes respectively as follows $R_{k}=\left(r_{i j}^{(k)}\right)_{5 \times 4}(k=1,2,3)$ which are recorded in
Tables 2-4 respectively.

Table 2. Linguistic assessing matrix by first expert

\begin{tabular}{|c|c|c|c|c|}
\hline & $\mathrm{G}_{1}$ & $\mathrm{G}_{2}$ & $\mathrm{G}_{3}$ & $\mathrm{G}_{4}$ \\
\hline$A_{1}$ & $\mathrm{~L}$ & $\mathrm{MH}$ & $\mathrm{M}$ & $\mathrm{VL}$ \\
\hline$A_{2}$ & $\mathrm{ML}$ & $\mathrm{L}$ & $\mathrm{VL}$ & $\mathrm{MH}$ \\
\hline$A_{3}$ & $\mathrm{ML}$ & $\mathrm{MH}$ & $\mathrm{M}$ & $\mathrm{M}$ \\
\hline$A_{4}$ & $\mathrm{H}$ & $\mathrm{VH}$ & $\mathrm{VH}$ & $\mathrm{ML}$ \\
\hline$A_{5}$ & $\mathrm{MH}$ & $\mathrm{M}$ & $\mathrm{L}$ & $\mathrm{L}$ \\
\hline
\end{tabular}

Table 3. Linguistic assessing matrix by second expert

\begin{tabular}{|c|c|c|c|c|}
\hline & $\mathrm{G}_{1}$ & $\mathrm{G}_{2}$ & $\mathrm{G}_{3}$ & $\mathrm{G}_{4}$ \\
\hline$A_{1}$ & $\mathrm{MH}$ & $\mathrm{MH}$ & $\mathrm{M}$ & $\mathrm{H}$ \\
\hline$A_{2}$ & $\mathrm{~L}$ & $\mathrm{M}$ & $\mathrm{VL}$ & $\mathrm{M}$ \\
\hline$A_{3}$ & $\mathrm{H}$ & $\mathrm{ML}$ & $\mathrm{M}$ & $\mathrm{ML}$ \\
\hline$A_{4}$ & $\mathrm{VH}$ & $\mathrm{MH}$ & $\mathrm{H}$ & $\mathrm{VH}$ \\
\hline$A_{5}$ & $\mathrm{VL}$ & $\mathrm{H}$ & $\mathrm{ML}$ & $\mathrm{MH}$ \\
\hline
\end{tabular}

Table 4. Linguistic assessing matrix by third expert

\begin{tabular}{|c|c|c|c|c|}
\hline & $\mathrm{G}_{1}$ & $\mathrm{G}_{2}$ & $\mathrm{G}_{3}$ & $\mathrm{G}_{4}$ \\
\hline$A_{1}$ & $\mathrm{H}$ & $\mathrm{M}$ & $\mathrm{ML}$ & $\mathrm{H}$ \\
\hline$A_{2}$ & $\mathrm{~L}$ & $\mathrm{MH}$ & $\mathrm{H}$ & $\mathrm{L}$ \\
\hline$A_{3}$ & $\mathrm{MH}$ & $\mathrm{L}$ & $\mathrm{M}$ & $\mathrm{ML}$ \\
\hline$A_{4}$ & $\mathrm{H}$ & $\mathrm{M}$ & $\mathrm{VH}$ & $\mathrm{MH}$ \\
\hline$A_{5}$ & $\mathrm{ML}$ & $\mathrm{ML}$ & $\mathrm{MH}$ & $\mathrm{H}$ \\
\hline
\end{tabular}

Following that, the developed approach is utilized to assess financial management performance of five possible enterprises.

Step 1. Transform the linguistic decision matrixes which are recorded in Tables 2-4 into 2TLPF decision matrix. The results are recorded in Tables 5-7. 
Table 5. The assessing matrix with 2 TLPFNs by first expert

\begin{tabular}{|c|c|c|c|c|}
\hline & $\mathrm{G}_{1}$ & $\mathrm{G}_{2}$ & $\mathrm{G}_{3}$ & $\mathrm{G}_{4}$ \\
\hline$A_{1}$ & $\left\{\left(s_{1}, 0\right),\left(s_{5}, 0\right)\right\}$ & $\left\{\left(s_{4}, 0\right),\left(s_{2}, 0\right)\right\}$ & $\left\{\left(s_{3}, 0\right),\left(s_{3}, 0\right)\right\}$ & $\left\{\left(s_{0}, 0\right),\left(s_{6}, 0\right)\right\}$ \\
\hline$A_{2}$ & $\left\{\left(s_{2}, 0\right),\left(s_{4}, 0\right)\right\}$ & $\left\{\left(s_{1}, 0\right),\left(s_{5}, 0\right)\right\}$ & $\left\{\left(s_{0}, 0\right),\left(s_{6}, 0\right)\right\}$ & $\left\{\left(s_{4}, 0\right),\left(s_{2}, 0\right)\right\}$ \\
\hline$A_{3}$ & $\left\{\left(s_{2}, 0\right),\left(s_{4}, 0\right)\right\}$ & $\left\{\left(s_{4}, 0\right),\left(s_{2}, 0\right)\right\}$ & $\left\{\left(s_{3}, 0\right),\left(s_{3}, 0\right)\right\}$ & $\left\{\left(s_{3}, 0\right),\left(s_{3}, 0\right)\right\}$ \\
\hline$A_{4}$ & $\left\{\left(s_{5}, 0\right),\left(s_{1}, 0\right)\right\}$ & $\left\{\left(s_{6}, 0\right),\left(s_{0}, 0\right)\right\}$ & $\left\{\left(s_{6}, 0\right),\left(s_{0}, 0\right)\right\}$ & $\left\{\left(s_{2}, 0\right),\left(s_{4}, 0\right)\right\}$ \\
\hline$A_{5}$ & $\left\{\left(s_{4}, 0\right),\left(s_{2}, 0\right)\right\}$ & $\left\{\left(s_{3}, 0\right),\left(s_{3}, 0\right)\right\}$ & $\left\{\left(s_{1}, 0\right),\left(s_{5}, 0\right)\right\}$ & $\left\{\left(s_{1}, 0\right),\left(s_{5}, 0\right)\right\}$ \\
\hline
\end{tabular}

Table 6. The assessing matrix with 2TLPFNs by second expert

\begin{tabular}{|c|c|c|c|c|}
\hline & $\mathrm{G}_{1}$ & $\mathrm{G}_{2}$ & $\mathrm{G}_{3}$ & $\mathrm{G}_{4}$ \\
\hline$A_{1}$ & $\left\{\left(s_{4}, 0\right),\left(s_{2}, 0\right)\right\}$ & $\left\{\left(s_{4}, 0\right),\left(s_{2}, 0\right)\right\}$ & $\left\{\left(s_{3}, 0\right),\left(s_{3}, 0\right)\right\}$ & $\left\{\left(s_{5}, 0\right),\left(s_{1}, 0\right)\right\}$ \\
\hline$A_{2}$ & $\left\{\left(s_{1}, 0\right),\left(s_{5}, 0\right)\right\}$ & $\left\{\left(s_{3}, 0\right),\left(s_{3}, 0\right)\right\}$ & $\left\{\left(s_{0}, 0\right),\left(s_{6}, 0\right)\right\}$ & $\left\{\left(s_{3}, 0\right),\left(s_{3}, 0\right)\right\}$ \\
\hline$A_{3}$ & $\left\{\left(s_{5}, 0\right),\left(s_{1}, 0\right)\right\}$ & $\left\{\left(s_{2}, 0\right),\left(s_{4}, 0\right)\right\}$ & $\left\{\left(s_{3}, 0\right),\left(s_{3}, 0\right)\right\}$ & $\left\{\left(s_{2}, 0\right),\left(s_{4}, 0\right)\right\}$ \\
\hline$A_{4}$ & $\left\{\left(s_{6}, 0\right),\left(s_{0}, 0\right)\right\}$ & $\left\{\left(s_{4}, 0\right),\left(s_{2}, 0\right)\right\}$ & $\left\{\left(s_{5}, 0\right),\left(s_{1}, 0\right)\right\}$ & $\left\{\left(s_{6}, 0\right),\left(s_{0}, 0\right)\right\}$ \\
\hline$A_{5}$ & $\left\{\left(s_{0}, 0\right),\left(s_{6}, 0\right)\right\}$ & $\left\{\left(s_{5}, 0\right),\left(s_{1}, 0\right)\right\}$ & $\left\{\left(s_{2}, 0\right),\left(s_{4}, 0\right)\right\}$ & $\left\{\left(s_{4}, 0\right),\left(s_{2}, 0\right)\right\}$ \\
\hline
\end{tabular}

Table 7. The assessing matrix with 2 TLPFNs by third expert

\begin{tabular}{|c|c|c|c|c|}
\hline & $\mathrm{G}_{1}$ & $\mathrm{G}_{2}$ & $\mathrm{G}_{3}$ & $\mathrm{G}_{4}$ \\
\hline$A_{1}$ & $\left\{\left(s_{5}, 0\right),\left(s_{1}, 0\right)\right\}$ & $\left\{\left(s_{3}, 0\right),\left(s_{3}, 0\right)\right\}$ & $\left\{\left(s_{2}, 0\right),\left(s_{4}, 0\right)\right\}$ & $\left\{\left(s_{5}, 0\right),\left(s_{1}, 0\right)\right\}$ \\
\hline$A_{2}$ & $\left\{\left(s_{1}, 0\right),\left(s_{5}, 0\right)\right\}$ & $\left\{\left(s_{4}, 0\right),\left(s_{2}, 0\right)\right\}$ & $\left\{\left(s_{5}, 0\right),\left(s_{1}, 0\right)\right\}$ & $\left\{\left(s_{1}, 0\right),\left(s_{5}, 0\right)\right\}$ \\
\hline$A_{3}$ & $\left\{\left(s_{4}, 0\right),\left(s_{2}, 0\right)\right\}$ & $\left\{\left(s_{1}, 0\right),\left(s_{5}, 0\right)\right\}$ & $\left\{\left(s_{3}, 0\right),\left(s_{3}, 0\right)\right\}$ & $\left\{\left(s_{2}, 0\right),\left(s_{4}, 0\right)\right\}$ \\
\hline$A_{4}$ & $\left\{\left(s_{5}, 0\right),\left(s_{1}, 0\right)\right\}$ & $\left\{\left(s_{3}, 0\right),\left(s_{3}, 0\right)\right\}$ & $\left\{\left(s_{6}, 0\right),\left(s_{0}, 0\right)\right\}$ & $\left\{\left(s_{4}, 0\right),\left(s_{2}, 0\right)\right\}$ \\
\hline$A_{5}$ & $\left\{\left(s_{2}, 0\right),\left(s_{4}, 0\right)\right\}$ & $\left\{\left(s_{2}, 0\right),\left(s_{4}, 0\right)\right\}$ & $\left\{\left(s_{4}, 0\right),\left(s_{2}, 0\right)\right\}$ & $\left\{\left(s_{5}, 0\right),\left(s_{1}, 0\right)\right\}$ \\
\hline
\end{tabular}

Step 2. According to Tables 5-7 and Eq. (19), the experts' individual evaluations can be fused into the collective assessing matrix with 2TLPFNs (Table 8).

Table 8. Collective assessing matrix with 2TLPFNs

\begin{tabular}{|c|c|c|c|c|}
\hline & $\mathrm{G}_{1}$ & $\mathrm{G}_{2}$ & $\mathrm{G}_{3}$ & $\mathrm{G}_{4}$ \\
\hline$A_{1}$ & $\left\{\left(s_{4}, 0.16\right),\left(s_{2},-0.05\right)\right\}$ & $\left\{\left(s_{4},-0.24\right),\left(s_{2}, 0.26\right)\right\}$ & $\left\{\left(s_{3},-0.25\right),\left(s_{3}, 0.27\right)\right\}$ & $\left\{\left(s_{5},-0.30\right),\left(s_{1}, 0.43\right)\right\}$ \\
\hline$A_{2}$ & $\left\{\left(s_{1}, 0.27\right),\left(s_{5},-0.22\right)\right\}$ & $\left\{\left(s_{3}, 0.16\right),\left(s_{3},-0.06\right)\right\}$ & $\left\{\left(s_{3}, 0.28\right),\left(s_{4},-0.49\right)\right\}$ & $\left\{\left(s_{3},-0.08\right),\left(s_{3}, 0.22\right)\right\}$ \\
\hline
\end{tabular}


End of Table 8

\begin{tabular}{|c|c|c|c|c|}
\hline & $\mathrm{G}_{1}$ & $\mathrm{G}_{2}$ & $\mathrm{G}_{3}$ & $\mathrm{G}_{4}$ \\
\hline$A_{3}$ & $\left\{\left(s_{4}, 0.44\right),\left(s_{2},-0.38\right)\right\}$ & $\left\{\left(s_{2}, 0.47\right),\left(s_{4},-0.28\right)\right\}$ & $\left\{\left(s_{3}, 0.00\right),\left(s_{3}, 0.00\right)\right\}$ & $\left\{\left(s_{2}, 0.25\right),\left(s_{4},-0.22\right)\right\}$ \\
\hline$A_{4}$ & $\left\{\left(s_{6}, 0.00\right),\left(s_{0}, 0.00\right)\right\}$ & $\left\{\left(s_{6}, 0.00\right),\left(s_{0}, 0.00\right)\right\}$ & $\left\{\left(s_{6}, 0.00\right),\left(s_{0}, 0.00\right)\right\}$ & $\left\{\left(s_{6}, 0.00\right),\left(s_{0}, 0.00\right)\right\}$ \\
\hline$A_{5}$ & $\left\{\left(s_{2}, 0.26\right),\left(s_{4}, 0.26\right)\right\}$ & $\left\{\left(s_{4}, 0.23\right),\left(s_{2},-0.11\right)\right\}$ & $\left\{\left(s_{3},-0.22\right),\left(s_{3}, 0.40\right)\right\}$ & $\left\{\left(s_{4}, 0.16\right),\left(s_{2},-0.05\right)\right\}$ \\
\hline
\end{tabular}

Step 3. Compute the weighted assessing matrix with 2TLPFNs (Table 9).

Table 9. Collective weighted assessing matrix with 2TLPFNs

\begin{tabular}{|c|c|c|c|c|}
\hline & $\mathrm{G}_{1}$ & $\mathrm{G}_{2}$ & $\mathrm{G}_{3}$ & $\mathrm{G}_{4}$ \\
\hline$A_{1}$ & $\left\{\left(s_{2}, 0.10\right),\left(s_{5},-0.21\right)\right\}$ & $\left\{\left(s_{2}, 0.23\right),\left(s_{4}, 0.48\right)\right\}$ & $\left\{\left(s_{2},-0.20\right),\left(s_{5},-0.29\right)\right\}$ & $\left\{\left(s_{2},-0.20\right),\left(s_{5}, 0.20\right)\right\}$ \\
\hline$A_{2}$ & $\left\{\left(s_{1},-0.43\right),\left(s_{6},-0.27\right)\right\}$ & $\left\{\left(s_{2},-0.17\right),\left(s_{5},-0.15\right)\right\}$ & $\left\{\left(s_{2}, 0.18\right),\left(s_{5},-0.16\right)\right\}$ & $\left\{\left(s_{1},-0.02\right),\left(s_{6},-0.36\right)\right\}$ \\
\hline$A_{3}$ & $\left\{\left(s_{2}, 0.30\right),\left(s_{5},-0.38\right)\right\}$ & $\left\{\left(s_{1}, 0.39\right),\left(s_{5}, 0.20\right)\right\}$ & $\left\{\left(s_{2},-0.02\right),\left(s_{5},-0.45\right)\right\}$ & $\left\{\left(s_{1},-0.26\right),\left(s_{6},-0.27\right)\right\}$ \\
\hline$A_{4}$ & $\left\{\left(s_{6}, 0.00\right),\left(s_{0}, 0.00\right)\right\}$ & $\left\{\left(s_{6}, 0.00\right),\left(s_{0}, 0.00\right)\right\}$ & $\left\{\left(s_{6}, 0.00\right),\left(s_{0}, 0.00\right)\right\}$ & $\left\{\left(s_{6}, 0.00\right),\left(s_{0}, 0.00\right)\right\}$ \\
\hline$A_{5}$ & $\left\{\left(s_{1}, 0.04\right),\left(s_{5},-0.40\right)\right\}$ & $\left\{\left(s_{3},-0.41\right),\left(s_{4}, 0.24\right)\right\}$ & $\left\{\left(s_{2},-0.18\right),\left(s_{5},-0.22\right)\right\}$ & $\left\{\left(s_{2},-0.49\right),\left(s_{5}, 0.36\right)\right\}$ \\
\hline
\end{tabular}

Step 4. Obtain the NIS by Eq. (22).The calculating results are recorded in Table 10.

Table 10. NIS with 2TLPFNs

\begin{tabular}{|c|c|c|c|}
\hline $\mathrm{G}_{1}$ & $\mathrm{G}_{2}$ & $\mathrm{G}_{3}$ & $\mathrm{G}_{4}$ \\
\hline$\left\{\left(s_{1},-0.43\right),\left(s_{6},-0.27\right)\right\}$ & $\left\{\left(s_{1}, 0.39\right),\left(s_{5}, 0.20\right)\right\}$ & $\left\{\left(s_{2}, 0.18\right),\left(s_{5},-0.16\right)\right\}$ & $\left\{\left(s_{1},-0.26\right),\left(s_{6},-0.27\right)\right\}$ \\
\hline
\end{tabular}

Step 5. Compute the $E D_{i}$ and $H D_{i}$ :

$$
\begin{aligned}
& E D_{1}=0.4915, E D_{2}=0.1406, E D_{3}=0.2749, E D_{4}=3.4141, E D_{5}=0.3384, \\
& H D_{1}=0.4766, H D_{2}=0.1292, H D_{3}=0.2690, H D_{4}=3.4098, H D_{5}=0.3240 .
\end{aligned}
$$

Step 6. Compute the $R A$ matrix (Table 11).

Table 11. Relative assessment matrix (RA)

\begin{tabular}{|c|c|c|c|c|c|}
\hline & $A_{1}$ & $A_{2}$ & $A_{3}$ & $A_{4}$ & $A_{5}$ \\
\hline$A_{1}$ & 0.0000 & 0.6869 & 0.4183 & -5.8602 & 0.2913 \\
\hline$A_{2}$ & -0.7132 & 0.0000 & -0.2800 & -6.5584 & -0.4069 \\
\hline$A_{3}$ & -0.4391 & 0.2627 & 0.0000 & -6.2844 & -0.1329 \\
\hline$A_{4}$ & 5.8410 & 6.5427 & 6.2741 & 0.0000 & 6.1472 \\
\hline$A_{5}$ & -0.3206 & 0.3812 & 0.1126 & -6.1658 & 0.0000 \\
\hline
\end{tabular}


Step 7. Compute the value of $A S_{i}$ by utilizing Eq. (28).

$$
A S_{1}=-4.4637, A S_{2}=-7.9585, A S_{3}=-6.5937, A S_{4}=24.8050, A S_{5}=-5.9926 .
$$

Step 8. In terms of the computing results of $A S_{i}$, all the alternatives can be ranked. Evidently, the order is $A_{4}>A_{1}>A_{5}>A_{3}>A_{2}$ and $A_{4}$ is the best one among five alternatives.

To indicate this method's validity, it is compared with the 2TLPF-TODIM method's result. The order of 2TLPF-TODIM is also $A_{4}>A_{1}>A_{5}>A_{3}>A_{2}$. As can be seen, the 2TLPFCODAS method's ranking result is totally consistent with 2TLPF-TODIM method. What's more, two distance formulas' combination is used in 2TLPF-CODAS method, which is more exact than the single one.

\section{Conclusions}

Financial management performance evaluation has a significant effect on the identifying an investment chance. Because this process can be regarded as a MAGDM issue, it is necessary to utilize an efficient MAGDM method for it. Besides, since the group decision-making process is within uncertain environment, it makes this assessment complex. In this paper, the expanding CODAS method has been developed to tackle MAGDM issues under 2TLPFNs. The weighted Euclidean and weighted Hamming distances of 2TLPFNs have been employed to decide the alternatives' desirability with regard to a negative-ideal solution. Also, we extend the crisp CODAS method utilizing the linguistic variables which are defined by 2TLPFNs. In the developed 2TLPF-CODAS method, the application of an example of financial management performance assessment problem is given. The comparative analysis demonstrates that the 2TLPF-CODAS method is effective and practical with 2TLPF-TODIM method. For further researches, the proposed method's application will be conducted in many other unpredictable and ambiguous environments.

\section{Acknowledgements}

This paper is supported by the National Natural Science Foundation of China (No. 71571128) and the Humanities and Social Sciences Foundation of Ministry of Education of the People's Republic of China (14XJCZH002, 16YJA840008).

\section{References}

Atanassov, K. T. (1986). Intuitionistic fuzzy sets. Fuzzy Sets and Systems, 20(1), 87-96. https://doi.org/10.1016/S0165-0114(86)80034-3

Badi, I., Ballem, M. A., \& Shetwan, A. (2018). Site selection of desalination plant in Libya by using combinative distance-based assessment (CODAS) method. International Journal for Quality Research, 12, 609-624.

Deng, X. M., \& Gao, H. (2019). TODIM method for multiple attribute decision making with 2-tuple linguistic Pythagorean fuzzy information. Journal of Intelligent \& Fuzzy Systems, 37(2), 1769-1780. https://doi.org/10.3233/JIFS-179240 
Deng, X. M., Wang, J., Wei, G. W., \& Lu, M. (2018a). Models for multiple attribute decision making with some 2-tuple linguistic Pythagorean fuzzy hamy mean operators. Mathematics, 6(11), 236. https://doi.org/10.3390/math6110236

Deng, X. M., Wei, G. W., Gao, H., \& Wang, J. (2018b). Models for safety assessment of construction project with some 2-tuple linguistic Pythagorean fuzzy Bonferroni mean operators. IEEE Access, 6, 52105-52137. https://doi.org/10.1109/ACCESS.2018.2869414

Erdogan, S. A., Saparauskas, J., \& Turskis, Z. (2019). A multi-criteria decision-making model to choose the best option for sustainable construction management. Sustainability, 11(8), 2239. https://doi.org/10.3390/su11082239

Garg, H. (2016). A new generalized Pythagorean fuzzy information aggregation using Einstein Operations and its application to decision making. International Journal of Intelligent Systems, 31(9), 886-920. https://doi.org/10.1002/int.21809

Garg, H. (2017a). Confidence levels based Pythagorean fuzzy aggregation operators and its application to decision-making process. Computational and Mathematical Organization Theory, 23(4), 546-571. https://doi.org/10.1007/s10588-017-9242-8

Garg, H. (2017b). A new improved score function of an interval-valued Pythagorean fuzzy set based topsis method. International Journal for Uncertainty Quantification, 7(5), 463-474. https://doi.org/10.1615/Int.J.UncertaintyQuantification.2017020197

Ghorabaee, M. K., Amiri, M., Zavadskas, E. K., Hooshmand, R., \& Antuchevičienè, J. (2018). Fuzzy extension of the CODAS method for multi-criteria market segment evaluation. Journal of Business Economics \& Management, 18(1), 1-19. https://doi.org/10.3846/16111699.2016.1278559

Herrera, F., \& Martinez, L. (2001a). The 2-tuple linguistic computational model. Advantages of its linguistic description, accuracy and consistency. International Journal of Uncertainty Fuzziness and Knowledge-Based Systems, 9(supp. 01), 33-48. https://doi.org/10.1142/S0218488501000971

Herrera, F., \& Martinez, L. (2001b). A model based on linguistic 2-tuples for dealing with multigranular hierarchical linguistic contexts in multi-expert decision-making. Ieee Transactions on Systems Man and Cybernetics Part B-Cybernetics, 31(2), 227-234. https://doi.org/10.1109/3477.915345

Keshavarz Ghorabaee, M., Zavadskas, E. K., Turskis, Z., \& Antucheviciene, J. (2016). A new combinative distance-based assessment (Codas) method for multi-criteria decision-making. Economic Computation \& Economic Cybernetics Studies \& Research, 50(3), 25-44.

Khan, M. S. A., Abdullah, S., Ali, A., Amin, F., \& Hussain, F. (2019). Pythagorean hesitant fuzzy Choquet integral aggregation operators and their application to multi-attribute decision-making. Soft Computing, 23, 251-267. https://doi.org/10.1007/s00500-018-3592-0

Li, Z. X., \& Lu, M. (2019). Some novel similarity and distance and measures of Pythagorean fuzzy sets and their applications. Journal of Intelligent \& Fuzzy Systems, 37(2), 1781-1799. https://doi.org/10.3233/JIFS-179241

Li, Z. X., Wei, G. W., \& Lu, M. (2018). Pythagorean fuzzy Hamy mean operators in multiple attribute group decision making and their application to supplier selection. Symmetry, 10(10), 505. https://doi.org/10.3390/sym10100505

Lu, J. P., Tang, X. Y., Wei, G. W., C. Wei, C., \& Wei, Y. (2019). Bidirectional project method for dual hesitant Pythagorean fuzzy multiple attribute decision-making and their application to performance assessment of new rural construction. International Journal of Intelligent Systems, 34(8), 1920-1934. https://doi.org/10.1002/int.22126

Pamucar, D., Badi, I., Sanja, K., \& Obradovic, R. (2018). A novel approach for the selection of powergeneration technology using a linguistic neutrosophic CODAS method: A case study in Libya. Energies, 11(9), 2489. https://doi.org/10.3390/en11092489 
Panchal, D., Chatterjee, P., Shukla, R. K., Choudhury, T., \& Tamosaitiene, J. (2017). Integrated fuzzy AHP-Codas framework for maintenance decision in urea fertilizer industry. Economic Computation and Economic Cybernetics Studies and Research, 51(3), 179-196.

Peng, X. D., \& Yang, Y. (2015). Some results for Pythagorean Fuzzy sets. International Journal of Intelligent Systems, 30(11), 1133-1160. https://doi.org/10.1002/int.21738

Reformat, M. Z., \& Yager, R. R. (2014). Suggesting recommendations using Pythagorean Fuzzy sets illustrated using netflix movie data. In A. Laurent, O. Strauss, B. BouchonMeunier, \& R. R. Yager (Eds.), Information processing and management of uncertainty in knowledge-based systems, Pt I (Vol. 442, pp. 546-556) Springer. https://doi.org/10.1007/978-3-319-08795-5_56

Ren, P. J., Xu, Z. S., \& Gou, X. J. (2016). Pythagorean fuzzy TODIM approach to multi-criteria decision making. Applied Soft Computing, 42, 246-259. https://doi.org/10.1016/j.asoc.2015.12.020

Roy, J., Sharma, H. K., Kar, S., Zavadskas, E. K., \& Saparauskas, J. (2019). An extended COPRAS model for multi-criteria decision-making problems and its application in web-based hotel evaluation and selection. Economic Research-Ekonomska Istrazivanja, 32(1), 219-253. https://doi.org/10.1080/1331677X.2018.1543054

Tabatabaei, M. H., Amiri, M., Firouzabadi, S., Ghahremanloo, M., Keshavarz-Ghorabaee, M., \& Saparauskas, J. (2019). A new group decision-making model based on bwm and its application to managerial problems. Transformations in Business \& Economics, 18, 197-214.

Wang, J., Gao, H., \& Lu, M. (2019a). Approaches to strategic supplier selection under interval neutrosophic environment. Journal of Intelligent \& Fuzzy Systems, 37(2), 1707-1730. https://doi.org/10.3233/JIFS-179235

Wang, J., Gao, H., \& Wei, G. W. (2019b). The generalized Dice similarity measures for Pythagorean fuzzy multiple attribute group decision making. International Journal of Intelligent Systems, 34(6), 1158-1183. https://doi.org/10.1002/int.22090

Wang, R. (2019). Research on the application of the financial investment risk appraisal models with some interval number muirhead mean operators. Journal of Intelligent \& Fuzzy Systems, 37(2), 1741-1752. https://doi.org/10.3233/JIFS-179237

Wei, G. W. (2019a). 2-tuple intuitionistic fuzzy linguistic aggregation operators in multiple attribute decision making. Iranian Journal of Fuzzy Systems, 16(4), 159-174.

Wei, G. W. (2019b). The generalized dice similarity measures for multiple attribute decision making with hesitant fuzzy linguistic information. Economic Research-Ekonomska Istrazivanja, 32(1), 1498-1520. https://doi.org/10.1080/1331677X.2019.1637765

Wei, G. W. (2019c). Pythagorean fuzzy hamacher power aggregation operators in multiple attribute decision making. Fundamenta Informaticae, 166(1), 57-85. https://doi.org/10.3233/FI-2019-1794

Wei, G. W., Wang, J., Wei, C., Wei, Y., \& Zhang, Y. (2019a). Dual hesitant Pythagorean fuzzy hamy mean operators in multiple attribute decision making. IEEE Access, 7, 86697-86716. https://doi.org/10.1109/ACCESS.2019.2924974

Wei, G. W., Wang, R., Wang, J., Wei, C., \& Zhang, Y. (2019b). Methods for evaluating the technological innovation capability for the high-tech enterprises with generalized interval neutrosophic number Bonferroni mean operators. IEEE Access, 7, 86473-86492. https://doi.org/10.1109/ACCESS.2019.2925702

Wu, L. P., Gao, H., \& Wei, C. (2019a). VIKOR method for financing risk assessment of rural tourism projects under interval-valued intuitionistic fuzzy environment. Journal of Intelligent \& Fuzzy Systems, 37(2), 2001-2008. https://doi.org/10.3233/JIFS-179262

Wu, L. P., Wang, J., \& Gao, H. (2019b). Models for competiveness evaluation of tourist destination with some interval-valued intuitionistic fuzzy Hamy mean operators. Journal of Intelligent and Fuzzy Systems, 36(6), 5693-5709. https://doi.org/10.3233/JIFS-181545 
Wu, L. P., Wei, G. W., Gao, H., \& Wei, Y. (2018). Some interval-valued intuitionistic fuzzy Dombi Hamy mean operators and their application for evaluating the elderly tourism service quality in tourism destination. Mathematics, 6(12), 294. https://doi.org/10.3390/math6120294

Zadeh, L. A. (1965). Fuzzy Sets. Information and Control, 8(3), 338-356. https://doi.org/10.1016/S0019-9958(65)90241-X

Zeb, A., Khan, M. S. A., \& Ibrar, M. (2019). Approaches to multi-attribute decision making with risk preference under extended Pythagorean fuzzy environment. Journal of Intelligent \& Fuzzy Systems, 36(1), 325-335. https://doi.org/10.3233/JIFS-181385

Zeng, S. Z., Chen, J. P., \& Li, X. S. (2016). A hybrid method for Pythagorean fuzzy multiple-criteria decision making. International Journal of Information Technology \& Decision Making, 15(2), 403-422. https://doi.org/10.1142/S0219622016500012

Zhang, C., Li, D. Y., \& Ren, R. (2016). Pythagorean fuzzy multigranulation rough set over two universes and its applications in Merger and acquisition. International Journal of Intelligent Systems, 31(9), 921-943. https://doi.org/10.1002/int.21811

Zhang, Q. S., \& Jiang, S. Y. (2010). Relationships between entropy and similarity measure of intervalvalued intuitionistic fuzzy sets. International Journal of Intelligent Systems, 25(11), 1121-1140. https://doi.org/10.1002/int.20442

Zhang, R. T., Wang, J., Zhu, X. M., Xia, M. M., \& Yu, M. (2017). Some generalized Pythagorean fuzzy Bonferroni mean aggregation operators with their application to multiattribute group decisionmaking. Complexity, 2017, 5937376. https://doi.org/10.1155/2017/5937376

Zhang, X. L. (2016). Multicriteria Pythagorean fuzzy decision analysis: A hierarchical QUALIFLEX approach with the closeness index-based ranking methods. Information Sciences, 330, 104-124. https://doi.org/10.1016/j.ins.2015.10.012

Zhu, L., Liang, X. F., Wang, L., \& Wu, X. R. (2018). Generalized pythagorean fuzzy point operators and their application in multi-attributes decision making. Journal of Intelligent \& Fuzzy Systems, 35(2), 1407-1418. https://doi.org/10.3233/JIFS-169683 